\title{
Interaction of ultrarelativistic electron and proton bunches with dense plasmas
}

\author{
A. A. Rukhadze ${ }^{1, *}$ and S. P. Sadykova ${ }^{2, \dagger}$ \\ ${ }^{1}$ Prokhorov General Physics Institute, Russian Academy of Sciences, Vavilov Street 38, Moscow 19991, Russia \\ ${ }^{2}$ Humboldt-Universität zu Berlin, Newtonstraße 15, Berlin 12489, Germany
}

(Received 6 January 2012; published 23 April 2012)

\begin{abstract}
Here we discuss the possibility of employment of ultrarelativistic monoenergetic electron and proton bunches for generation of high plasma wakefields in dense plasmas due to Cherenkov resonance plasmabunch interaction. We estimate the maximum amplitude of such a wakefield and minimum plasma, bunch lengths at which the maximum amplitude can be generated at the given plasma and bunch parameters.
\end{abstract}

DOI: 10.1103/PhysRevSTAB.15.041302

PACS numbers: 52.40.Mj, 41.60.Bq, 52.35.Qz

\section{INTRODUCTION}

Proposals for generating high wakefields by nonrelativistic electron bunches propagating through plasma were first made in 1949 [1,2]. High-energy bunch electrons generate the wake plasma wave (wake) in such a way that the energy from a bunch of electrons is transferred to the plasma wave through Cherenkov resonance radiation producing high electric fields (wakefields). Later, it was shown that the relativistic electron bunches can generate plasma waves with high relativistic phase velocity including the transverse electromagnetic waves efficiently radiated out of plasma [3]. This led to the birth of a new applied science-high-power pulse relativistic superhighfrequency (SHF) electronics enabling one to produce the high-power pulse broadened SHF amplifiers and generators on a base of such plasma-bunch systems [4,5].

The idea to accelerate the charged particles in a plasma medium using collective plasma fields belongs to Budker, Veksler, and Fainberg [6-8]. Later on, another acceleration scheme using a time-shifted sequence of bunched highenergy electrons injected into a cold plasma was proposed when the late-coming electron bunch riding on the wave, produced by the first-coming bunch, at a proper phase will be boosted to a higher energy [9]. In recent experiments at the Stanford Linear Accelerator Center it was shown that an energy gain of more than $42 \mathrm{GeV}$ was achieved in a meter long plasma-wakefield accelerator, driven by a $42 \mathrm{GeV}$ electron beam [10]. For a detailed review about the modern status of this research field, we would like to refer the reader to [11].

Recently, the possibility of generation of high-power wakefields (proton-bunch-driven plasma-wakefield

\footnotetext{
*rukh@fpl.gpi.ru

Corresponding author.

saltanat@physik.hu-berlin.de

Published by the American Physical Society under the terms of the Creative Commons Attribution 3.0 License. Further distribution of this work must maintain attribution to the author(s) and the published article's title, journal citation, and DOI.
}

acceleration) of terawatt amplitude using the ultrarelativistic proton bunches was introduced $[12,13]$.

In the present work, this idea along with the employment of ultrarelativistic electron bunch is discussed at the qualitative level. Namely, we make an estimation of plasma parameters, maximum amplitude of the generated wakefield when the ultrarelativistic electron and proton bunches are employed and plasma, bunch lengths at which the maximum amplitude of the wakefield can be gained.

\section{ULTRARELATIVISTIC ELECTRON BUNCH}

Let us start our analysis with the ultrarelativistic monoenergetic electron bunch of density $n_{b}$ and velocity $\vec{u}$, noting that

$$
\gamma=\frac{1}{\sqrt{1-u^{2} / c^{2}}} \gg 1
$$

Such a bunch interacts with the cold (no thermal motion) isotropic plasma being in a rest of density $n_{p} \gg n_{b}$ and generates the plane wave $E=E_{0} \exp (-i \omega t+i \vec{k} \cdot \vec{r})$; here $\omega$ is the frequency and $\vec{k}$ is the wave vector. We choose an axis $Z$ directed along the velocity of the bunch $\vec{u}$, and put the external field as absent. ${ }^{2}$ The dispersion relation in the laboratory frame of reference (plasma in a rest) describing the amplification of a plasma wave (longitudinal transverse) with the help of a bunch can be written as follows [3]:

$$
\begin{gathered}
\left(k^{2} c^{2}-\omega^{2}+\omega_{p}^{2}+\omega_{b}^{2} \gamma^{-1}\right)\left(1-\frac{\omega_{p}^{2}}{\omega^{2}}-\frac{\omega_{b}^{2} \gamma^{-3}}{\left(\omega-k_{z} u\right)^{2}}\right) \\
-\frac{k_{\perp}^{2} u^{2}}{\omega^{2}} \frac{\omega_{p}^{2} \omega_{b}^{2} \gamma^{-1}}{\left(\omega-k_{z} u\right)^{2}}=0 .
\end{gathered}
$$

Here $\quad \omega_{p}=\sqrt{4 \pi e^{2} n_{p} / m}, \quad \omega_{b}=\sqrt{4 \pi e^{2} n_{b} / m} \quad$ are Langmuir plasma electron and bunch frequencies

\footnotetext{
${ }^{2}$ In ultrarelativistic bunches, bunch divergence can be neglected because when the electron bunch is ejected into the dense plasma, within the time $t \sim 1 / \omega_{p}$ the neutralization of the bunch charge occurs prohibiting the bunch divergence [4].
} 
respectively (in Gaussian Unit System units) with $n_{p}, n_{b}$ being the plasma and bunch densities, $k_{z}$ and $k_{\perp}$ are the longitudinal (directed along the velocity of the bunch $\vec{u}$ ) and transverse components of the wave vector $\vec{k}$. We put no boundary conditions on the plasma-bunch system. This relation represents the plane wave solution of Maxwell's equations for a plasma-bunch system making use of Lorentz transformations for the electric field $\vec{E}$ and dielectric permittivity $\varepsilon(\omega, \vec{k})$. The plasma ion terms were neglected in the derivation, i.e., only the interaction of the electron bunch with the high-frequency plasma oscillations is considered. The plasma wave emerging from an initial fluctuation amplifies with the time and the system becomes unstable. The bunch terms are the most significant in the frequency range of Cherenkov resonance, i.e., when the bunch speed $u$ coincides with the phase velocity of the plasma oscillations $\omega_{p} / k$.

Hence, the general solution of Eq. (2) $\omega(\vec{k})$ at $\omega \approx$ $k_{z} u \approx \omega_{p}$ with the positive imaginary part $(\Im m \omega>0)$ will be the following:

$$
\begin{aligned}
& \omega=k_{z} u(1+\delta)=\omega_{p}(1+\delta) \\
& \delta=\frac{-1+i \sqrt{3}}{2}\left(\frac{n_{b}}{2 n_{p}} \frac{1}{\gamma}\right)^{1 / 3}\left(1-\frac{u^{2}}{c^{2}} \frac{\omega_{p}^{2}}{\omega_{p}^{2}+k_{\perp}^{2} u^{2}}\right)^{1 / 3} .
\end{aligned}
$$

Equation (3) shows that the oscillations with the fre-

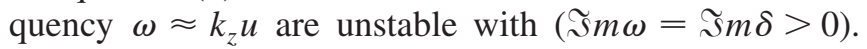
From Eq. (3) follows that at $\Re e \delta<0-u>\omega / k_{z}$ meaning that the bunch electrons overtake the wave and transfer part of their kinetic energy to the wave potential energy producing the high wakefields. Since the value $\Im m \delta$ (increment), determining the temporary growth of the plasma wave amplitude generated by the bunch, depends on $k_{\perp}$, then in this way it depends on the transverse bunch size $R_{0}$ (bunch radius), $k_{\perp} \sim 1 / R_{0}$.

For a start we would like to be far away from the concrete magnitudes of the concrete system parameters and consider two opposite limiting cases.

(A) In a case of dense plasma when

$$
\omega_{p}^{2} \gg k_{\perp}^{2} u^{2} \sim u^{2} / R_{0}^{2} .
$$

Then, the value $\delta$ is given by the expression

$$
\delta=\frac{-1+i \sqrt{3}}{2}\left(\frac{n_{b}}{2 n_{p}}\right)^{1 / 3} \frac{1}{\gamma} .
$$

(B) In a case of rare plasma when the inverse to (4) inequality is satisfied then the value $\delta$ becomes

$$
\delta=\frac{-1+i \sqrt{3}}{2}\left(\frac{n_{b}}{2 n_{p}} \frac{1}{\gamma}\right)^{1 / 3} .
$$

Let us outline again that while deriving Eqs. (3)-(6) we took into account that value $\delta$ is negligibly small which is sustained by the inequalities $n_{b} \ll n_{p}$ and $\gamma^{2} \gg 1$. Here, we should point out that the bunch radius also influences the constraint for plasma density (4), consequently the increments $\delta$ (5) and (6).

We would like to stress that for the development of instability and plasma wave growth it is necessary that the following constraint for a plasma length must be satisfied:

$$
L>\frac{u}{\delta \omega_{p}}
$$

being at the same time limited due to the bunch divergence to the following constraint:

$$
L<\frac{u \sqrt{\gamma}}{\omega_{b}}=\frac{u \sqrt{\gamma}}{\left(n_{b} / n_{p}\right)^{1 / 2} \omega_{p}} .
$$

Here the imaginary part of $\delta(\Im m \delta)$ is considered. The bunch length is chosen to be equal to the plasma length and is measured in the laboratory frame of reference.

Let us now estimate the maximum amplitude of the generated by the bunch wakefield, so called the amplitude of saturated instability. For this, we choose the wake as a frame of reference moving with the speed $\omega / k_{z}$ with respect to the laboratory frame of reference. In accordance with Lorentz transformations, the speed of bunch electrons in the chosen frame will be

$$
u_{1}=-\frac{u \delta \gamma^{2}}{1-\frac{2 u^{2}}{c^{2}} \delta \gamma^{2}}=-\frac{u \delta \gamma^{2}}{1-2 \delta\left(\gamma^{2}-1\right)}
$$

Here the real part of $\delta(\Re e \delta$ ) is considered. From Eq. (8) follows that in the relativistic limit when $\left(|\delta| \gamma^{2} \ll 1\right)$ this speed is small $u_{1} \approx-u \delta \gamma^{2} \ll u$, whereas in the ultrarelativistic limit $\left(|\delta| \gamma^{2} \gg 1\right)$ the speed is high $u_{1} \approx u / 2$. We would like to point out that the ultrarelativistic speed of the electron bunch (8) is still relativistic in the wake frame of reference compared to that considered earlier which was nonrelativistic [14].

It is obvious that the saturation of instability can occur when the kinetic energy of bunch electrons, in the wake frame of reference, will become less than the saturation amplitude of the potential of the plasma wake, generated by this bunch in dense plasma, which is measured in the same frame of reference. In this case the bunch electrons get trapped by the wake, i.e., there will be no relative motion between the bunch electrons and the wake, thus, no energy exchange between the bunch and wake occurs, the bunch and the wake become stationary. Hence, the stationary saturation amplitude of the plasma wake potential can be obtained from the following equation:

$$
\frac{e \Phi_{0}}{m c^{2}}=\frac{1}{\gamma}\left\{\frac{1}{\sqrt{1-\frac{u^{2} \delta^{2} \gamma^{4}}{c^{2}\left[1-2 \delta\left(\gamma^{2}-1\right)\right]^{2}}}}-1\right\} .
$$

Here $\Phi_{0} \gamma$ is measured in the wake frame of reference. In the relativistic limit when $\left(\delta \gamma^{2} \ll 1\right)$ we will get the result 
already obtained in [14] for dense plasma, Eq. (5), whereas in the ultrarelativistic limit when $\left(\delta \gamma^{2} \gg 1\right)$ we obtain

$$
\frac{e \Phi_{0}}{m c^{2}} \approx \frac{0.154}{\gamma}
$$

Here $\Phi_{0}$ is measured in the laboratory frame of reference. Equation (10) leads to the following conclusion: the amplitude of the potential of the plasma wake generated by the electron bunch is dependent on the relativity factor $\Phi_{0}=$ $0.154 m c^{2} /(e \gamma)$. This is demonstrated in Fig. 1. We can observe a maximum $\Phi_{0 \max } \simeq 770 \mathrm{~V}$ for dense plasma at $n_{p}=10^{16} \mathrm{~cm}^{-3}, \gamma_{0} \simeq 24$ and $\Phi_{0 \text { max }} \simeq 15.5 \times 10^{3} \mathrm{~V} \simeq$ $15.5 \mathrm{kV}$ for rare plasma at $n_{p}=6 \times 10^{12} \mathrm{~cm}^{-3}, \gamma_{0} \simeq$ 2.56; see the Appendix. It is quite easy to estimate the

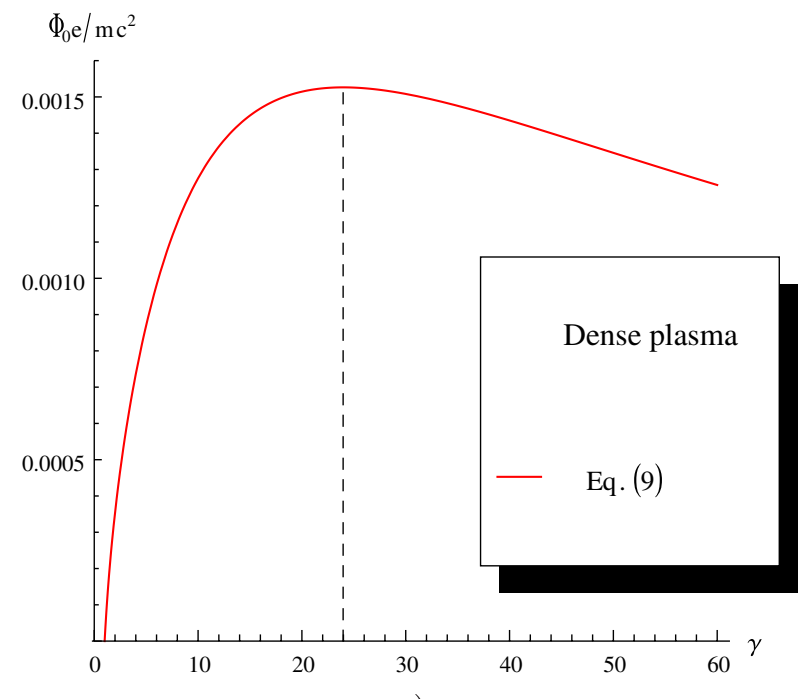

a)

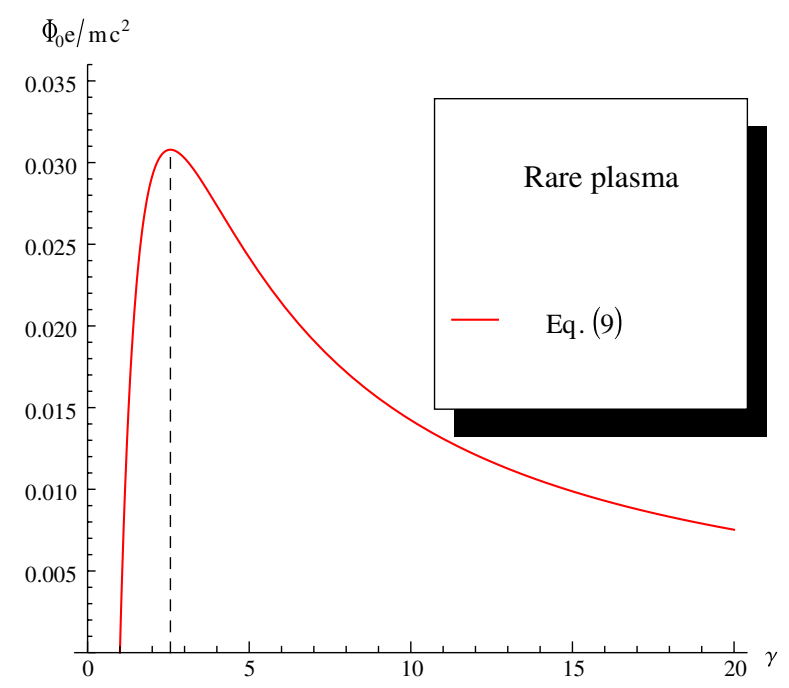

c) saturation amplitude of the electric field of the wake generated by the bunch. For this in Eq. (9), one needs to take into account that $E_{0} \sim k \Phi_{0}$ leading to the following equation for the relative energy density of the electrostatic field:

$$
\frac{E_{0}^{2}}{8 \pi n_{p} m c^{2} \gamma} \simeq \frac{c^{2}}{u^{2}} \frac{1}{2 \gamma^{3}}\left\{\frac{1}{\sqrt{1-\frac{u^{2} \delta^{2} \gamma^{4}}{c^{2}\left[1-2 \delta\left(\gamma^{2}-1\right)\right]^{2}}}}-1\right\}^{2} .
$$

In the relativistic limit when $\left(\delta \gamma^{2} \ll 1\right)$ this equation turns into the result obtained in [14] for dense plasma, Eq. (5), whereas in the ultrarelativistic limit when $\left(\delta \gamma^{2} \gg 1\right)$ we get

$$
\frac{E_{0}^{2}}{8 \pi n_{p} m c^{2} \gamma} \simeq \frac{0.012}{\gamma^{3}}
$$

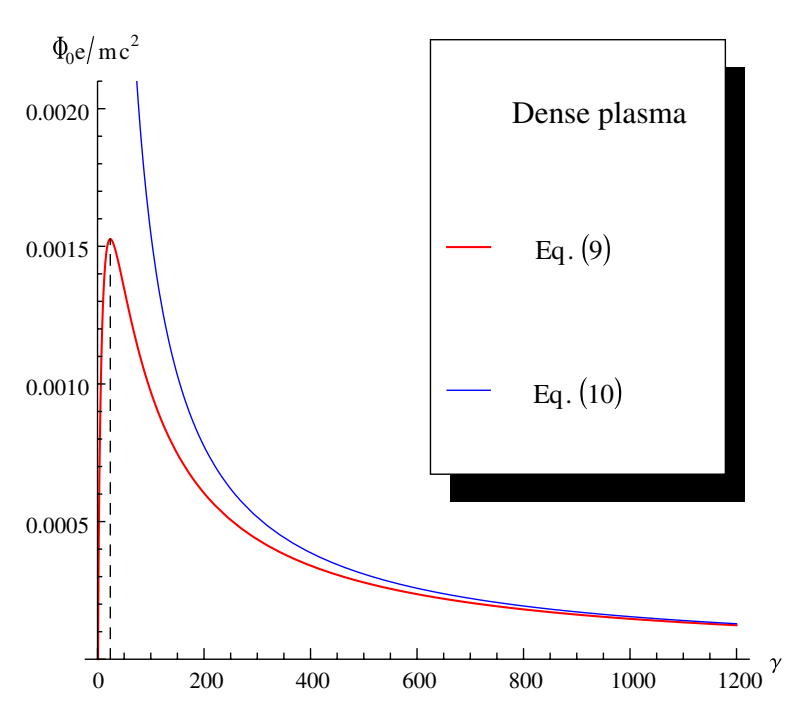

b)

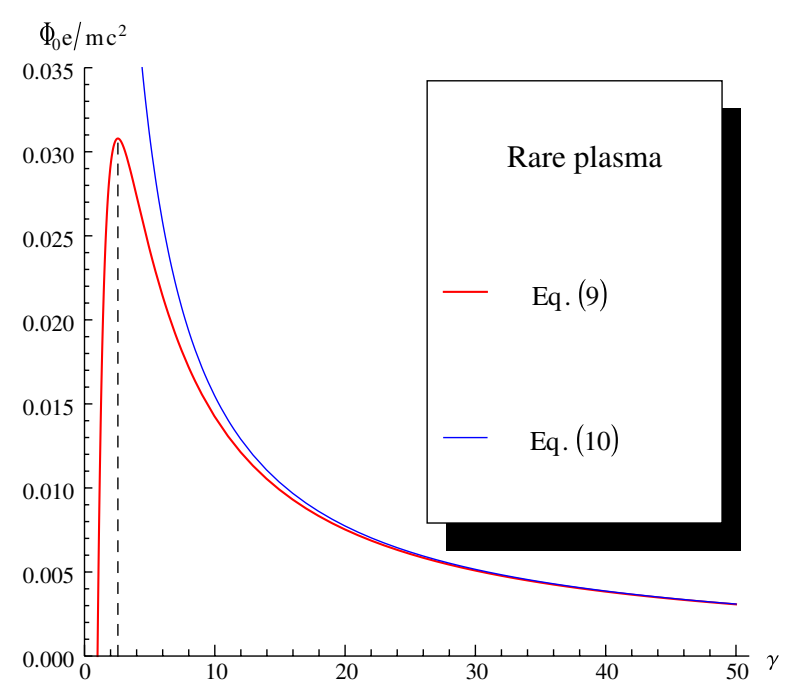

d)

FIG. 1. (a) The relative stationary saturation amplitude of the plasma wake potential generated by the ultrarelativistic electron bunch Eq. (9) for (a), (b) dense (5) at $n_{b}=2 \times 10^{12} \mathrm{~cm}^{-3}, n_{p}=10^{16} \mathrm{~cm}^{-3}, \gamma_{0}=24$ and (c), (d) rare (6) plasmas at $n_{b}=2 \times 10^{12} \mathrm{~cm}^{-3}$, $n_{p}=6 \times 10^{12} \mathrm{~cm}^{-3}, \gamma_{0}=2.6$. In (b) and (d) the relative stationary saturation amplitude in the ultrarelativistic limit (10) is presented for comparison. 
We can readily see that with an increase of relativity the amplitude of the electric field of the wake generated by the electron bunch in its turn is dependent on the relativity $\gamma$ as it is shown in Fig. 1 and is also defined in the ultrarelativistic limit by the plasma density $n_{p}$, $E_{0} \simeq 0.024 \sqrt{4 \pi n_{p} m c^{2}} / \gamma$. For example, for dense plasma at $\gamma_{0} \simeq 24, \quad n_{p}=10^{16} \mathrm{~cm}^{-3}$, we have $E_{0 \max } \simeq$ $1.45 \times 10^{5} \mathrm{~V} / \mathrm{cm} \simeq 14.5 \mathrm{MV} / \mathrm{m}$, whereas for rare plasma at $n_{p}=6 \times 10^{12} \mathrm{~cm}^{-3}, \quad \gamma_{0} \simeq 2.56$ we have $E_{0 \max } \simeq$ $7.2 \times 10^{4} \mathrm{~V} / \mathrm{cm} \simeq 7.2 \mathrm{MV} / \mathrm{m}$.

\section{ULTRARELATIVISTIC PROTON BUNCH}

As it has been mentioned above, in works $[12,13]$ the possibility of generation of high-power wakefields (protonbunch-driven plasma-wakefield acceleration) of terawatt amplitude using the ultrarelativistic proton bunches of giga-electron-volt energy was introduced. The idea is that to slow the protons down using the plasma wakefield is much harder than the electrons and as a result the significantly higher wakefield amplitude can be gained in comparison with that produced by the electron bunch.

The dispersion relation (2) can be easily generalized for the case of plasma-proton-bunch interaction. Correspondingly, we need to make the substitution $n_{b} \rightarrow$ $n_{b i}$ ( $n_{b}$-electron bunch density), i.e., substitute the $n_{b}$ by the proton bunch density $n_{b i}$ multiplied by the ratio of electron mass $m$ to the ion mass $M, m / M$.

Having solved Eq. (2), we write the solution generalizing Eq. (3) for the proton bunch down:

$$
\begin{aligned}
\omega & =k_{z} u\left(1+\delta_{1}\right)=\omega_{p}\left(1+\delta_{1}\right) \\
\delta_{1} & =\frac{-1+i \sqrt{3}}{2}\left(\frac{n_{b}}{2 n_{p}} \frac{m}{M} \frac{1}{\gamma}\right)^{1 / 3}\left(1-\frac{u^{2}}{c^{2}} \frac{\omega_{p}^{2}}{\omega_{p}^{2}+k_{\perp}^{2} u^{2}}\right)^{1 / 3} .
\end{aligned}
$$

Here $\delta_{1}$ differs from $\delta$, from Eq. (3), in $n_{b} \rightarrow n_{b i} \frac{m}{M}$. Since the increment $\delta_{1}$, determining the temporary growth of the plasma wave amplitude generated by the proton bunch, depends on $k_{\perp}$, then in this way it depends on the transverse bunch size $R_{0}$ (bunch radius), $k_{\perp} \sim 1 / R_{0}$.

(A) In the dense plasma when the constraint (4) is satisfied [compare with (5)], the value $\delta_{1}$ takes the following view:

$$
\delta_{1}=\frac{-1+i \sqrt{3}}{2}\left(\frac{n_{b i}}{2 n_{p}} \frac{m}{M}\right)^{1 / 3} \frac{1}{\gamma} .
$$

(B) In the rare plasma when the inverse to (4) constraint is satisfied, then the value $\delta_{1}$ [compare with (6)] becomes

$$
\delta_{1}=\frac{-1+i \sqrt{3}}{2}\left(\frac{n_{b i}}{2 n_{p}} \frac{m}{M} \frac{1}{\gamma}\right)^{1 / 3} .
$$

Here, we should point out again that the bunch radius also influences the constraint for plasma density (4), consequently the increments $\delta$ (14) and (15).
We would like to point out again that as in the case for the electron bunch the development of proton bunch instability and plasma wake growth can occur when the following constraint similar to Eq. (7) for a plasma length must be satisfied:

$$
L>\frac{u}{\delta_{1} \omega_{p}} .
$$

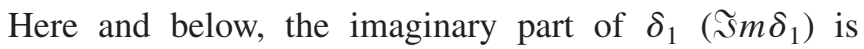
considered. However, the plasma length must be limited as well in order to neglect the proton bunch divergence due to the proton charges repulsion:

$$
L<\frac{u \sqrt{\gamma}}{\omega_{b i}}=\frac{u \sqrt{\gamma}}{\left(\frac{n_{b i}}{n_{p}} \frac{m}{M}\right)^{1 / 2} \omega_{p}} .
$$

The bunch length is chosen to be equal to the plasma length and is measured in the laboratory frame of reference.

Similarly, we estimate the maximum amplitude of the generated by the proton bunch wakefield, so called the amplitude of saturated instability. For this, we choose again the wake as a frame of reference moving with the speed $\omega / k_{z}$ with respect to the laboratory frame of reference. In this case, the speed of bunch protons in this frame [compare with (8)] will be

$$
u_{1}=-\frac{u \delta_{1} \gamma^{2}}{1-\frac{2 u^{2}}{c^{2}} \delta_{1} \gamma^{2}}=-\frac{u \delta_{1} \gamma^{2}}{1-2 \delta_{1}\left(\gamma^{2}-1\right)} .
$$

Here the real part of $\delta\left(\Re e \delta_{1}\right)$ is considered. From Eq. (17) we get the following result: in the relativistic limit when $\left(\left|\delta_{1}\right| \gamma^{2} \ll 1\right)$ this speed is small $u_{1} \simeq-u \delta_{1} \gamma^{2} \ll u$, whereas in the ultrarelativistic limit $\left(\left|\delta_{1}\right| \gamma^{2} \gg 1\right)$ the speed is high $u_{1} \simeq u / 2$. We would like to point out that the ultrarelativistic speed of the proton bunch (17) is still relativistic in the wake frame of reference. We would like to note that ultrarelativity of the proton bunch can be reached at much higher proton energies: $\gamma^{2}$ should be at least 1 order of magnitude higher compared to that of the electron bunch. For example, when we take for electron bunch $-n_{b} / n_{p} \sim 10^{-3}$, the ultrarelativity can be reached at $\gamma \geq 10$ (electron bunch energy is higher than $5 \mathrm{MeV}$ ), whereas for the proton bunch at the same density ratio-only at $\gamma \geq 50$ (proton bunch energy should be higher than $44 \mathrm{GeV}$ ).

By analogy with Eq. (9), the stationary saturation amplitude of the plasma wake potential, produced by the proton bunch in dense plasma, can be obtained from the following equation:

$$
\frac{e \Phi_{0}}{M c^{2}}=\frac{1}{\gamma}\left\{\frac{1}{\sqrt{1-\frac{u^{2} \delta_{1}^{2} \gamma^{4}}{c^{2}\left[1-2 \delta_{1}\left(\gamma^{2}-1\right)\right]^{2}}}}-1\right\}
$$

Here $\Phi_{0} \gamma$ is measured in the wake frame of reference. Correspondingly, in the ultrarelativistic limit when $\delta_{1} \gamma^{2} \gg 1$, we get an estimate similar to Eq. (10): 


$$
\frac{e \Phi_{0}}{M c^{2}} \approx \frac{0.154}{\gamma}
$$

Here $\Phi_{0}$ is measured in the laboratory frame of reference. Equation (19) leads to the following conclusion: the saturation amplitude of the potential of the plasma wake generated by the ultrarelativistic proton bunch is also dependent on the relativity factor $\Phi_{0}=0.154 M c^{2} /(e \gamma)$. This can be observed in Fig. 2. As we can see it possesses a maximum. We have estimated it for dense plasma at $n_{p}=$ $10^{16} \mathrm{~cm}^{-3}, \gamma_{0} \simeq 289$ leading to $\Phi_{0 \max } \simeq 1.1 \times 10^{5} \mathrm{~V} \simeq$ $0.1 \mathrm{MV}$ and rare plasma at $n_{p}=6 \times 10^{12} \mathrm{~cm}^{-3}, \gamma_{0} \simeq$ 11.6 leading to $\Phi_{0 \max } \simeq 5.74 \times 10^{6} \mathrm{~V} \simeq 5.74 \mathrm{MV}$, see the Appendix.

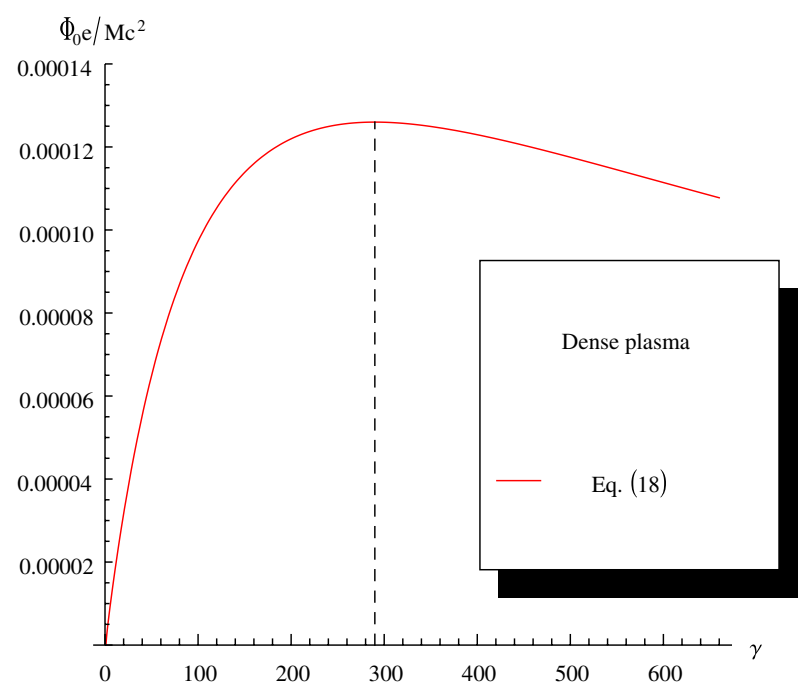

a)

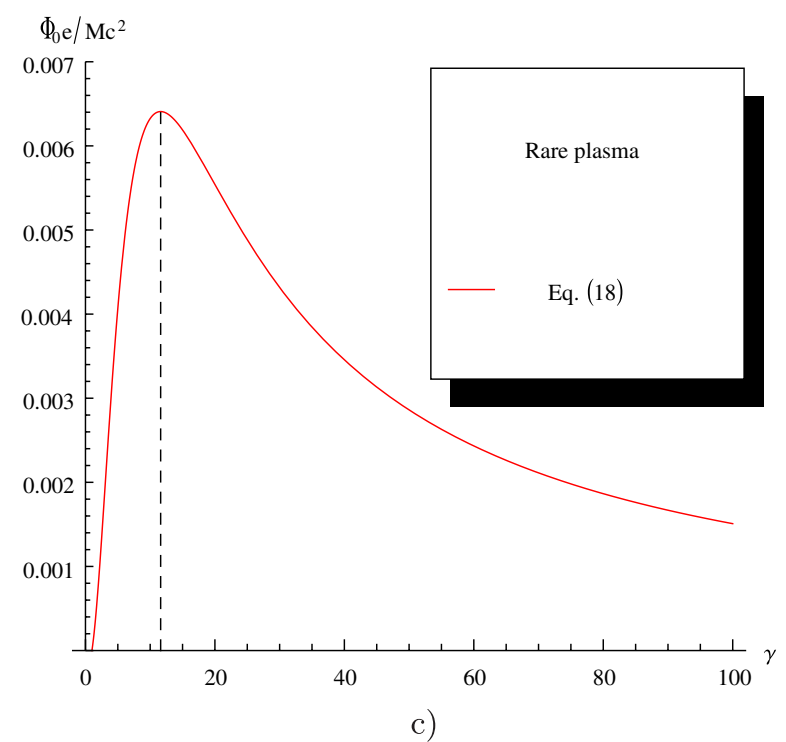

Again, it is quite easy to estimate the saturation amplitude of the electric field of the wake generated by the proton bunch. For this in Eq. (18), taking into account that $E_{0} \sim k \Phi_{0}$, we obtain the following equation for the relative energy density of the electrostatic field:

$\frac{E_{0}^{2}}{8 \pi n_{p} M c^{2} \gamma} \simeq \frac{c^{2}}{u^{2}} \frac{1}{2 \gamma^{3}}\left\{\frac{1}{\sqrt{1-\frac{u^{2} \delta_{1}^{2} \gamma^{4}}{c^{2}\left[1-2 \delta_{1}\left(\gamma^{2}-1\right)\right]^{2}}}}-1\right\}^{2}$

leading in the ultrarelativistic limit for the proton bunch when $\delta_{1} \gamma^{2} \gg 1$ to [compare with (12)]

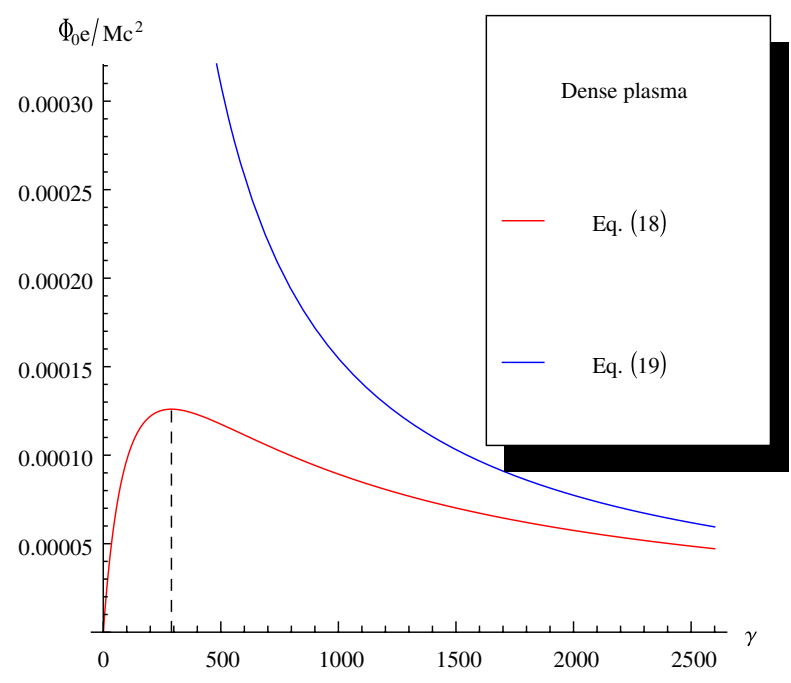

b)

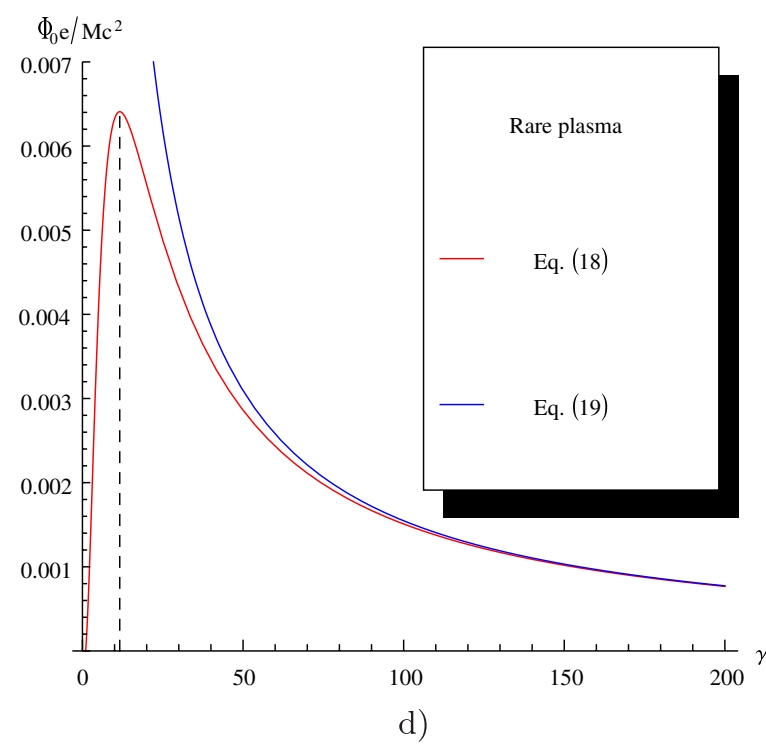

FIG. 2. (a) The relative stationary saturation amplitude of the plasma wake potential generated by the ultrarelativistic proton bunch Eq. (18) for (a), (b) dense (14) at $n_{b}=2 \times 10^{12} \mathrm{~cm}^{-3}, n_{p}=10^{16} \mathrm{~cm}^{-3}, \gamma_{0}=289$ and (c), (d) rare (15) plasmas at $n_{b}=$ $2 \times 10^{12} \mathrm{~cm}^{-3}, n_{p}=6 \times 10^{12} \mathrm{~cm}^{-3}, \gamma_{0}=11.6$. In (b) and (d) the relative stationary saturation amplitude in the ultrarelativistic limit (19) is presented for comparison. 


$$
\frac{E_{0}^{2}}{8 \pi n_{p} M c^{2} \gamma} \simeq \frac{0.012}{\gamma^{3}}
$$

We can readily see that the saturation amplitude of the potential as well as that of the electric field of the wake generated by the proton bunch in its turn is defined by the relativity factor as it is shown in Fig. 2 and plasma density $n_{p}$, namely $E_{0}=0.024 \sqrt{4 \pi n_{p} M c^{2}} / \gamma$. For example, for dense plasma at $n_{p}=10^{16} \mathrm{~cm}^{-3}, \gamma_{0} \simeq 289$ we have $E_{0 \text { max }} \simeq 5 \times 10^{5} \mathrm{~V} / \mathrm{cm} \simeq 50 \mathrm{MV} / \mathrm{m}$, for rare plasma at $n_{p}=6 \times 10^{12} \mathrm{~cm}^{-3}, \quad \gamma_{0} \simeq 11.6$ we have $E_{0 \max } \simeq$ $6.3 \times 10^{5} \mathrm{~V} / \mathrm{cm} \simeq 63 \mathrm{MV} / \mathrm{m}$.

\section{RESULTS AND DISCUSSIONS}

In the present work for the first time the analytical problem of interaction of ultrarelativistic electron and proton bunches with dense plasmas $\left(n_{p} \gg n_{b}\right)$ has been solved. These bunches remain relativistic in the frame of reference of wakes generated by these bunches compared to those considered earlier which were nonrelativistic.

On the basis of the conducted analysis of the resonance Cherenkov interaction of the ultrarelativistic monoenergetic electron as well as proton bunches with plasma, we can make the following conclusion: the wake amplitude growth produced by the bunches gets saturated with an increase of bunch energy at a not quite high level. The saturation amplitude of the electric wakefield possesses a maximum in dependence on the relativity factor $\gamma$ and should be tuned in accordance with the constraints for Cherenkov resonance in either dense or rare plasmas, plasma and bunch densities. This amplitude can be increased by increasing the plasma and bunch densities. The highest amplitude of the electric wakefield produced by the electron bunch can be generated in dense plasma at $n_{p}=10^{16} \mathrm{~cm}^{-3}$ and is of order $14.5 \mathrm{MV} / \mathrm{m}$, whereas that produced by the proton bunch is the highest in the rare plasma at $n_{p}=6 \times 10^{12} \mathrm{~cm}^{-3}$ and is of order $63 \mathrm{MV} / \mathrm{m}$. These magnitudes are less than those gained with the help of contemporary quite powerful pulse lasers $\left(10^{15} \mathrm{~W} / \mathrm{cm}^{2}\right)$.

As a reader may have noticed, the estimated magnitudes are different from those observed in the SLAC experiment, where the energy doubling of $42 \mathrm{GeV}$ electrons, constituting approximately $1 \%$ of all beam electrons, in a plasmawakefield accelerator was detected [10]. The implied by authors peak accelerating field is $52 \mathrm{GeV} / \mathrm{m}$. We presume that this discrepancy is due to the following: we consider the resonance Cherenkov interaction of the ultrarelativistic monoenergetic electron beam with plasma in a framework of the hydrodynamic model neglecting the thermal motion of plasma particles and plasma, bunch particles collisions [4], whereas in the SLAC experiment the nominally $42 \mathrm{GeV}$ beam with a correlated energy spread of $1.5 \mathrm{GeV}$ was employed, where, in our opinion, the kinetic bunch-plasma instabilities take place. Furthermore, the outlined above constraints should be taken into account as well.

In conclusion, it must be noticed that nonlinear plasma Langmuir wave is unstable and, as it was shown in [15], it breaks down when

$$
e \Phi_{0}>m c^{2} \gamma_{w}
$$

where $\gamma_{w}=1 / \sqrt{1-u_{\mathrm{ph}}^{2} / c^{2}}$ and $u_{\mathrm{ph}}$ is the wave phase velocity. In our case $u_{\mathrm{ph}}=u$ and $\gamma_{w}=\gamma$. From the results of our calculations follows that considered resonance Cherenkov instabilities arise for electron as well as for proton bunches when the plasma wake amplitudes are much less than the breakdown thresholds.

For further detailed research, we are planning to run simulations of the considered phenomena using the KARAT code [16].

\section{ACKNOWLEDGMENTS}

Here we consider the spatially unbounded plasma-bunch systems which can also be referred to as the spatially unbounded plasma-beam systems. S. P. Sadykova would like to express her gratitude to her father, P. S. Sadykov, for his financial support of the work and for being all the way the great moral support for her.

\section{APPENDIX: ESTIMATION OF THE MAXIMUM MAGNITUDE OF THE WAKEFIELD AND WAKE POTENTIAL}

In order to determine the maximum value of the $\Phi_{0}$ let us represent the Eq. (9) in the following way independent on the type of interaction either electron-plasma or protonplasma:

$$
\frac{e \Phi_{0}}{m c^{2}}=\left(\frac{\alpha}{Z}\right)^{1 / n}\left\{\frac{1+2 Z}{\sqrt{1+4 Z+3 Z^{2}}}-1\right\},
$$

where $Z=\delta_{\{, 1} \gamma^{2}=-\alpha \gamma^{n}, \quad n=1$ corresponds to the dense plasma, and $n=5 / 3$ - the rare plasma, $\alpha=$ $\frac{-1}{2}\left(\frac{n_{b i}}{2 n_{p}} \frac{m}{M}\right)^{1 / 3}$ for proton bunch and $\alpha=\frac{-1}{2}\left(\frac{n_{b}}{2 n_{p}}\right)^{1 / 3}$ for electron bunch.

Equation (A1) has a peak at $Z=0.55546$ for dense plasma $(n=1)$ and the parameters $\alpha$ and $\gamma$ should be changed according to the relation

$$
0.55546=\alpha \gamma .
$$

For rare plasma $(n=5 / 3)$ at $Z=1.35695$ we have

$$
1.35695=\alpha \gamma^{5 / 3} \text {. }
$$

These values correspond to the maxima of the wake potentials for the dense plasma

$$
\frac{e \Phi_{0 \max }}{\{M, m\} c^{2}}=-0.06576 \alpha
$$


and for the rare plasma

$$
\frac{e \Phi_{0 \max }}{\{M, m\} c^{2}}=-0.0618 \alpha^{3 / 5}
$$

Correspondingly, for the wakefields for the dense plasma

$$
\frac{E_{0 \max }}{\sqrt{\{M, m\} c^{2}}}=-0.06576 \alpha \sqrt{4 \pi n_{p}}
$$

and for the rare plasma

$$
\frac{E_{0 \max }}{\sqrt{\{M, m\} c^{2}}}=-0.0618 \alpha^{3 / 5} \sqrt{4 \pi n_{p}} .
$$

From Eqs. (A4)-(A7) follows that we should increase $\alpha$ in such a way that the constraints (4)-(6), (14), and (15), $\delta_{\{\}, 1} \ll 1, \gamma^{2} \gg 1$ are not violated. As one can readily see, the highest saturation amplitude of the plasma wake potential corresponds to the rare plasma, whereas the highest saturation wakefield can be generated in the dense plasma.

[1] A. I. Ahiezer and Ya. B. Fainberg, Dokl. Akad. Nauk SSSR 69, 555 (1949) [http://www.springerlink.com/ content/1063-7761].

[2] D. Bohm and E. Gross, Phys. Rev. 75, 1864(1949).

[3] A. A. Rukhadze, Zh. Tekh. Fiz. 31, 1236 (1961).

[4] A.F. Alexandrov, L.S. Bogdankevich, and A. A. Rukhadze, Principles of Plasma Electrodynamics (Springer, Heidelberg, 1984), pp. 167-170.
[5] M. V. Kuzelev and A. A. Rukhadze, Plasma Free Electron Lasers (Edition Frontier, Paris, 1995).

[6] G. I. Budker, in Proceedings of the CERN Symposium on High Energy Accelerators and Pion Physics (CERN, Geneva, 1956), Vol. 1, p. 68.

[7] V. I. Veksler, in Proceedings of the CERN Symposium on High Energy Accelerators and Pion Physics (Ref. [6]), Vol. 1, p. 80 .

[8] Ia. B. Fainberg, in Proceedings of the CERN Symposium on High Energy Accelerators and Pion Physics (Ref. [6]), Vol. 1, p. 84.

[9] P. Chen, J. M. Dawson, R. W. Huff, and T. Katsouleas, Phys. Rev. Lett. 54, 693 (1985).

[10] I. Blumenfeld et al., Nature (London) 445, 741 (2007).

[11] C. Joshi and M. Victor, New J. Phys. 12, 1 (2010).

[12] A. Caldwell, K. Lotov, A. Pukhov, and F. Simon, Nature Phys. 5, 363 (2009).

[13] N. Kumar, A. Pukhov, and K. Lotov, Phys. Rev. Lett. 104, 255003 (2010).

[14] R. I. Kovtun and A. A. Rukhadze, Zh. Eksp. Teor. Fiz. 58, 1709 (1970).

[15] A. I. Akhiezer, I. A. Akhiezer, R. V. Polovin, A. G. Sitenko, and K. N. Stepanov, Plasma Electrodynamics, V.1. Linear Theory, International Series of Monographs in Natural Philosophy, Vol. 68 (Pergamon Press, OxfordNew York, 1975); Plasma Electrodynamics, V.2. Nonlinear theory and fluctuations, International Series of Monographs in Natural Philosophy, Vol. 69 (Pergamon Press, Oxford-New York, 1975).

[16] V.P. Tarakanov, User's Manual for Code KARAT (Berkeley Research Associates, Inc., Springfield, VA, 1992). 